\title{
Use and Implications of a Shared, Forecasting Calendar
}

\author{
Joe Tullio $^{1}$ and Elizabeth D. Mynatt ${ }^{2}$ \\ ${ }^{1}$ Motorola Labs \\ Schaumburg, Illinois, USA \\ joe.tullio@motorola.com \\ ${ }^{2}$ Graphics, Visualization, and Usability Center \\ Georgia Institute of Technology \\ Atlanta, Georgia, USA \\ mynatt@cc.gatech.edu
}

\begin{abstract}
Changes in modern work environments, combined with advances in sensing and machine intelligence, have given rise to a new class of groupware applications that seeks to facilitate workplace communication through the prediction of future availability and/or location. We present the results of a four-month deployment of an experimental predictive calendar system in an academic setting. While participants appreciated several novel features of the system, most resisted adoption due to the uncertainty of its predictions, its effects on privacy and impression management, and accessibility issues. We present implications for designers who seek to incorporate forecasting components into their groupware tools using observations from the study.
\end{abstract}

Keywords: Groupware calendar system, evaluation, intelligent user interfaces, forecasting, communication, privacy.

\section{Introduction}

Informal communication remains a critical aspect of work. However, groupware tools that are used to facilitate and mediate informal communication are less useful when people become increasingly mobile or have more flexible schedules. In response, researchers have undertaken projects that make use of new advances in activity sensing and machine intelligence to arrive at inferences about current or future availability and/or location $[4,8,11,12,15]$. In most cases, they are intended to foster better informal communication in the workplace. To date, a great deal of progress has been made towards designing architectures for such systems and improving their performance through various representations and learning algorithms.

Researchers are presently in need of qualitative evaluations from real-world environments to guide the future design and deployment of predictive groupware systems. Such evaluations can provide insights beyond their feasibility, reliability, and accuracy. Important observations can be made about how forecasts of availability and presence meet objectives of facilitating both formal and informal communication while respecting privacy and social norms. 
In this paper, we use an augmented shared calendar system called Augur as the basis such a study. By incorporating user models and machine learning techniques, Augur enhances a traditional calendar interface with predictions of attendance at future events. In addition, Augur identifies events scheduled by multiple colleagues and displays these matches in the standard calendar view.

We present the results of a field study of Augur in an academic setting, examining how participants with varying job descriptions, working relationships, and existing scheduling practices used the system over a period of four months. We found that participants appreciated the event-matching feature and used it as a means of maintaining social and workplace awareness. We also found that attendance predictions benefited a subset of working relationships within our user population.

However, we also found that the uncertainty inherent in Augur's user models, combined with non-routine periods during the study, created additional privacy and impression management tasks that may have outweighed the benefits of forecasting for some users. These issues, combined with a lack of accessibility, resulted in limited adoption of Augur as a primary calendar application. This work nonetheless provides insights for future systems by recommending lightweight design elements to support calendars as social awareness tools, to institute privacy protections for third-parties, and to include additional controls for impression management.

\section{Related Work}

A number of existing research systems seek to predict the current and future states of their users. These systems can manage incoming electronic notifications [9] or phone calls [11] based on sensed activity, or can forecast states of interruptibility and availability $[2,4,5,10,13]$. While many of these systems have been deployed, there are few published results on the qualitative effects these systems have had on the work practices of their users. The research instead focuses on system development and refinement of the core predictive models or machine learning techniques.

Fogarty et al. augmented an instant messaging client with predictions of interruptibility and deployed it to several workgroups [5]. Through quantitative analysis of use logs, the researchers examined how predictive information affected use of the client, finding that estimates were primarily used to determine presence.

The motivations underlying most systems that forecast presence and availability are to streamline informal communication, a well-studied aspect of workplace collaboration [20]. For our purposes, we used a definition that defines communicative formality as a continuum along dimensions such as preset agenda, number of participants, and advance planning [14].

Groupware calendar systems represent an important class of tools for enabling this communication. Work by Palen [16] as well as work she performed with Grudin [7, 17] examined electronic calendar use at two large companies, finding that workers often browsed their colleagues' calendars to infer where they might be and when they might return to their offices. Mynatt and Tullio observe that if two coworkers share some events on their calendars, one of them can wait until one of those shared events takes place to "ambush" the other for an informal chat [15]. When a calendar includes conflicting events or infrequently attended events, however, its value as a reliable predictor of a person's true schedule is weakened. 


\section{The Augur Calendar System}

Augur is a web-based shared calendar system that predicts a person's attendance at their future events [19]. In addition, Augur identifies when the same event has been scheduled by multiple colleagues and displays these matched events on the user's calendar. Augur is designed to mitigate communication problems associated with poor calendar maintenance, inadequate firsthand knowledge of schedules, and low collocation by enabling a more reliable view of a colleague's calendar. By automatically synchronizing schedule data from PDAs or other desktop calendar applications, Augur does not force users to learn a new interface for creating and editing their schedules. Rather, it serves as a read-only service capable of displaying user calendars on most web browsers. Augur predicts attendance at future scheduled events by using manually-created Bayesian networks that encode the probabilistic relationships between various attributes of those events. These attributes include explicit properties such as start/end times and alarm settings as well as inferred properties such as event type (individual meeting, seminar, etc.) and building-level location. Inferred properties are classified using support-vector machines trained on labeled events. Lastly, the Bayesian models learn over time from self-reported attendance data, and attendance predictions are updated daily.

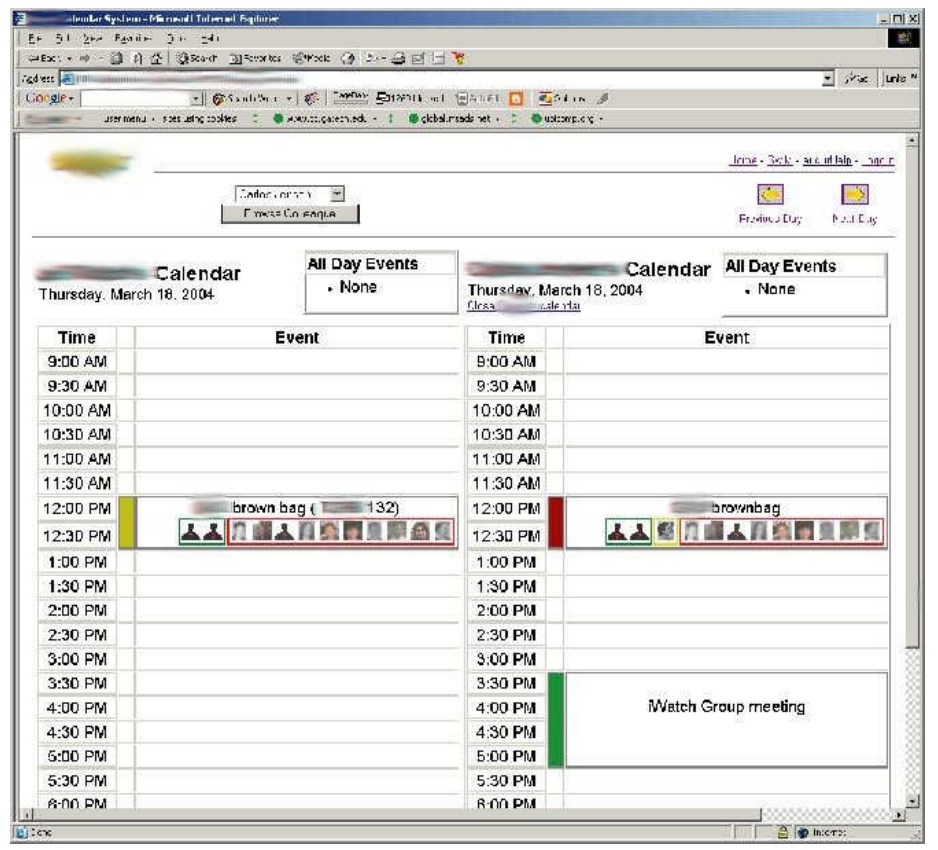

Fig. 1. The Augur calendar system. Two colleague calendars are shown side-by-side with the same event scheduled at noon. Icons within each event indicate colleagues who have also scheduled the event. Attendance predictions are shown as vertical bars next to each event, and as colored borders around colleague icons. 
A screen shot of the main Augur interface is shown in Figure 1. The standard tabular calendar format is used, but additional graphical elements encode the predictive information. Color-coded vertical bars indicate attendance likelihood for a given event, while thumbnail portraits inside the event's cell indicate which of the user's colleagues also have the event scheduled. Colored borders around these portraits indicate the likelihood of attendance by those colleagues. Color codes range from bright green for a high likelihood of attendance to bright red for a very low likelihood, with yellow being neutral. Clicking on a portrait allows a user to view that colleague's schedule alongside her own schedule. Access-control lists are used to control who can view another user's calendar.

\section{Method}

\subsection{Study Site}

We deployed Augur in an academic setting that offered a familiar set of tasks and relationships but made no guarantees that existing practices were compatible with the introduction of a groupware calendar system. To gauge the extent of technology use for the purposes of scheduling and communicating among students, professors, and staff, we conducted an email-based survey. We received responses from 33 faculty/staff and 70 graduate students representing all sub-areas of the department out of a total population of about 167 faculty/staff and 450 graduate students. Results of the survey showed that nearly $70 \%$ of respondents kept an electronic calendar, and that $30 \%$ were accustomed to sharing their calendar in some fashion. Calendar sharing strategies were diverse, with respondents using email, printouts, .plan files on Unix systems, web pages, and shared spreadsheets, to name a few. Some respondents used several of these strategies to increase their accessibility.

We determined that this setting would provide a challenging but rich environment for our study. Participants would be able to synchronize a diverse range of calendar artifacts with the system while retaining their existing methods. A variety of working relationships between students, professors, and administrative assistants could be examined to see how each of them benefited from predictive calendaring.

\subsection{Participants and Recruitment}

Twenty-seven participants were recruited from a university engineering department to share their calendar data, with 18 students, 8 professors, and one administrative staff member publishing their calendars. Participants were offered five dollars per week up to a total of $\$ 50$ for participating. They were asked to self-report their event attendance, to be willing to have their use of the system logged, and in some cases to participate in interviews several times throughout the study period. They were not required to use the system as a tool for their work. Also, 30 others volunteered to have "read-only" accounts that permitted them to browse others' calendars without contributing their own. The participants' relationships ranged from people who were physically or occupationally isolated from one other to students and advisors working closely in the same labs. 


\subsection{Study Structure}

The study period lasted approximately four months. We structured the study to first deploy a "plain" version of Augur that did not include predictions about attendance and co-scheduled events. After six weeks, the predictive features were enabled, and participants used this full version of Augur for the remainder of the study period. The intent of this structure was threefold. First, the initial six weeks of the study allowed participants to adjust to a common calendar infrastructure. Second, it allowed comparisons to be made on use of the system before and after the introduction of predictive features. Third, the attendance information collected early in the study allowed models to be trained prior to exposing the predictive features to participants.

Both qualitative and quantitative data collection was used during the course of the study. Logging routines captured logins and event views on Augur, and also archived predictions and old calendar information. Attendance data was collected by having participants complete a web-based form that reported whether each event was attended, missed to attend another event, or simply not attended.

We selected 13 of our participants (9 students, 4 faculty) to take part in four 40 minute interviews each. They were selected based on the diversity of relationships they had with other participants and the diversity of their existing scheduling habits. They were first interviewed prior to Augur's deployment to examine initial concerns about privacy, expectations for the system, and existing coordination practices. They were interviewed again after the "plain" version of Augur was deployed to gauge how this more traditional shared calendar was used. The third and fourth interviews took place after the introduction of predictive features in Augur.

To examine the use of Augur with respect to colleague relationships, interviewed participants selected up to three colleagues with calendars on the system and described their working relationships in terms of how often the two colleagues met, the degree of formality in those meetings, how much time they spent in the same location, the degree of schedule knowledge they had of one another, and how sufficient that knowledge was for conducting work together. We documented use for a total of 28 participant relationships, revisiting them in subsequent interviews to determine what benefits, if any, were being gained from using Augur.

\section{Results}

Augur was deployed for one academic semester. Figure 2 shows the number of events accessed from Augur over the course of the study period. Clearly, use tapered off somewhat as the study progressed, with gaps appearing during spring break (March 812) and the week of final exams (April 26-30). In this section, we examine use of Augur and identify which users and relationships tended to benefit from it.

\subsection{Overall Use}

For work-related communication, participants generally continued to rely primarily on their existing scheduling tools, the most popular being email and office visits. For those working fairly closely, tools were typically already in place to coordinate communication. In these cases, Augur was employed on an as-needed basis when 


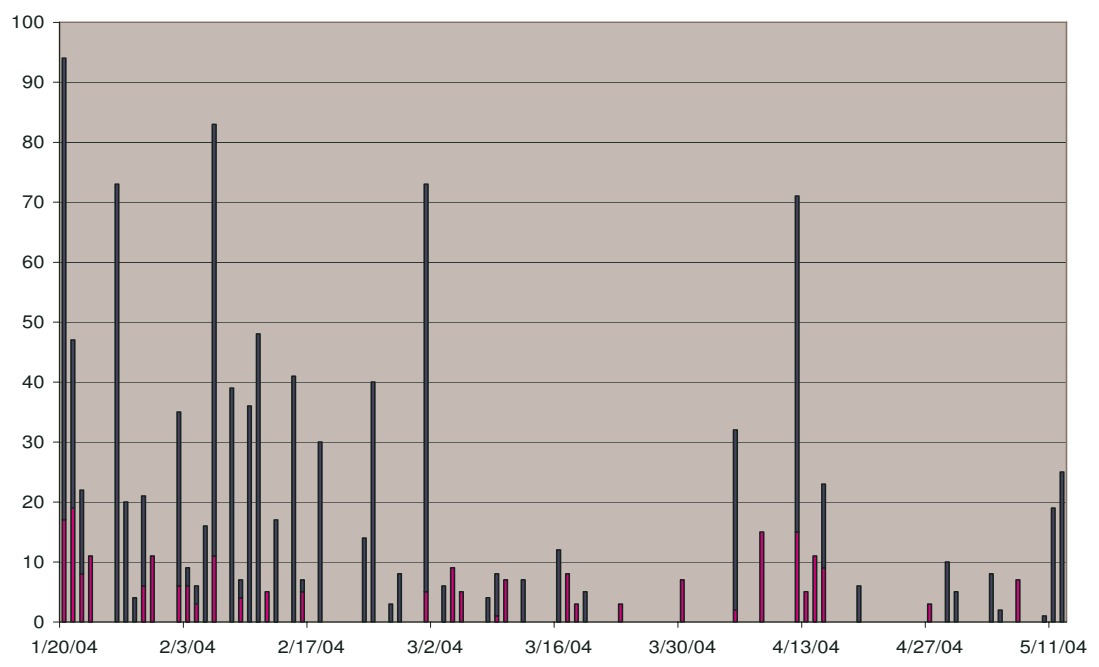

Fig. 2. Event accesses by date. Interviewed participants are shown in red.

these existing tools lacked sufficient detail. As one student stated: "I don't rely on it, but it does what I need it to do when I use it". As discussed below, however, Augur was more frequently used as a social awareness application.

By the end of the study, only two participants had adopted Augur as their primary calendar application. One, an administrator, had no other access to his superior's calendar, and the other, a student, publicized it to interested family and friends. In both of these cases, each participant had no previously existing way of sharing his calendar or browsing a desired calendar. Later, we discuss possible reasons as to why Augur was not more widely adopted.

\section{"Calendar Surfing"}

The most successfully supported and used activity on Augur was the practice of what one participant termed "calendar surfing". Almost half of the use instances (19) documented in interviews involved this activity. By embedding links to colleague calendars in its user interface, Augur lets users easily jump between, or "surf" these calendars when they share events with others. Participants most frequently mentioned using Augur in this fashion, usually stating no particular motive other than "curiosity", "out of boredom", or "for fun". Many times, however, these seemingly frivolous uses had work-related purposes behind them. When elaborating on these uses in interviews, we found that surfing behavior served several functions for indirectly sharing information between participants.

In keeping with Palen's findings, participants used Augur for information retrieval, adding events from other calendars that were not in their own schedules:

I just had my first great moment in Augur. I found an event in your calendar, a special talk that I want to attend. I just added it to my calendar.

Participants also browsed calendars to confirm their own intuitions about a particular person's schedule. One student was surprised that a professor's calendar 
was the "morass" she was expecting, while another student was skeptical of the accuracy of the events on his advisor's schedule.

Lastly, participants used the calendar links embedded in Augur's UI for social purposes, either to learn more about unfamiliar coworkers or to check in on existing workplace friends.

\section{Meeting scheduling}

Augur was also used as a regular shared calendar for tasks Palen has termed "calendar work" [16]. These tasks include meeting scheduling, orienting oneself throughout the day, and reminding. The second-most popular use of Augur among interviewed participants was for meeting scheduling, with participants using the calendar to locate free/busy times. No one cited attendance predictions as a resource they used for this task, with just the presence of events being enough to cause them to look elsewhere for open times on the calendar. Instead, the main benefit of Augur was to provide scheduling information for many colleagues that had previously been nonexistent or lacking in details.

\section{Finding another colleague}

Augur's attendance prediction feature was designed to allow colleagues to more easily locate one another for informal communication. Only three participants mentioned using Augur's predictions to explicitly find someone, with a subset of participants reporting "surfing" calendars to learn more about a colleague's likely schedule. Two of these instances involved student/advisor relationships, while one involved a staff member and his superior. As an example, one student spoke of an attempt to find his advisor:

I was trying to find out where his afternoon event was. I saw that the prediction was green (meaning likely attendance), so I didn't expect to see him in the lab then.

\subsection{Use Within Relationships}

\section{Social relationships and intermittent relationships}

Augur's predictions of attendance had little bearing on four primarily social relationships examined during the study. Interactions in these relationships tended to be fixed around unscheduled after-work events or a flexible lunchtime. Consequently, few uses of Augur were reported that concerned a specific pair of social colleagues. Only one instance of use, a session of calendar surfing, was reported within these relationships.

In addition, few uses were reported with to four relationships that revolved around intermittent, formal group meetings. The additional capabilities of Augur provided little benefit to them, since their working relationships are sufficiently distant that they can work purely from scheduled meetings. Thus, a system like Augur provides no benefit over a standard calendar application. The few uses that were reported were primarily out of curiosity over another colleague's schedule that had previously been a black box.

\section{Close colleagues}

Nine of the relationships we documented involve colleagues whose offices are physically collocated and who interact at least twice a week, indicating a greater 
degree of interdependence. Within these relationships, however, we noticed a disparity between those colleagues who are highly co-present and those who are not. We recorded equal amounts of use between both of these subgroups. For those colleagues who are less co-present, Augur provided a source of knowledge that acted as a substitute for the understanding normally shared by collocated colleagues.

In one student/advisor relationship, for example, the two colleagues work in the same lab space, but due to classes, meetings, and in/out times are typically not in the $\mathrm{lab}$ at the same time. Meetings between the two are a mix of both formal and informal interactions. On several occasions, the student would consult Augur to find a good time in the near future to catch his advisor in the lab for an informal chat.

The closest colleagues have a number of existing artifacts available for informing one another of their schedules, including paper printouts on doors and web-based calendars. However, nearly all of these artifacts are created at the start of the term and left untouched until the next one. Thus, they lack the detail of a more fastidiously maintained personal calendar, which is more likely to contain non-recurring, special events. Since Augur shares the personal calendar of its owner, it became a last resort resource when face-to-face channels and other calendar artifacts failed.

\section{Less close colleagues}

Colleagues who meet less frequently and whose offices are not necessarily in the same location comprise a second set of working relationships that are less close than those just described. These relationships are characterized by moderate copresence and meeting frequency, and a high degree of informality. Examples include graduate students and their thesis committee members, and labmates with fairly disparate research projects. Eleven of the relationships studied fell into this category, with use of Augur reported for five of them. We found that these five relationships all exhibited insufficient schedule knowledge for coordinating unplanned meetings.

As an example, two participants included a lab manager (an administrative position) and the professor who supervises the same lab. Their working relationship requires occasional interaction. They reside in different buildings, but have a standing meeting and intermittent email communication. The professor has a number of other responsibilities which frequently delay the standing meeting. Thus, the meeting has taken on more of an informal quality, as its timing is very flexible from week to week:

Last week was fairly typical. [The professor] missed our Friday meeting. I stopped by the office, he wasn't there. So then I went to another meeting in the same building. I came back and he was there.

The lab manager reported using Augur to determine when to make the 20-minute trip to the professor's building rather than using the oft-missed standing meeting time.

\section{Power relationships}

It is worth mentioning here that the goals of participants were somewhat different at the onset of the Augur study. With differing occupations came different power roles, whether superior/subordinate relationships or peer relationships.

It was clear that subordinates found more use for Augur than their superiors. This finding was consistent with prior reports of calendar use in industrial settings [6]. While interviewed students reported using Augur to find their advisors, thesis committee members, or instructors, interviewed professors reported participating in 
the system predominantly as a means to be found. Administrative assistants also welcomed the opportunity to see professors' calendars online, one of whom asked that the system continue to run after the study period had ended.

\subsection{Working with Uncertainty}

\section{Image concerns}

In adding predictive capabilities to Augur, we wanted to see how participants would react to shared information that was inherently uncertain and at times fallible. When participants mentioned Augur's predictions, it was often with respect to its effects on their image rather than its use in communication.

Concerns were voiced by one student even before Augur's deployment regarding control over the image presented of him to others: "I have control over my selfpresentation. I can fake other meetings to control my schedule". Several other participants, both students and professors, listed control over their calendars as the primary benefit of their prior method of scheduling.

Once predictions were introduced, several participants mentioned the potentially negative effects these predictions could have on their images, echoing the concerns over manually-assigned event priorities in work by Beard et al. [1]. For instance, a student was initially disturbed when he appeared not to attend a particular event, stating that he felt like "the system was taking attendance". He was relieved, however, when he saw that a professor was predicted not to attend the same event, and said "if there are enough people that don't attend, that's ok." One professor, appearing "likely" to attend a class he was teaching, wanted to appear "very likely".

We also received several emails concerning attendance reporting. Specifically, participants were concerned about the treatment of events that were either canceled in advance or otherwise disrupted (e.g., other parties in a meeting did not show up). We determined that these worries were due to issues of image. Participants did not want to look like they were doing a poor job of attending certain scheduled events when the reasons for not attending were beyond their control. According to one professor, "with certain weekly meetings, I would always go if they were happening".

Augur tended to overstate its predictions of how many users had scheduled the same events. Models of coscheduled events that had performed well for smaller research groups [19] were inadequate for our comparatively large user population due to multiple definitions for jargon, acronyms, and initials in event descriptions. These predictions were sometimes described as "decidedly weird" or puzzling. And while users were quick to notice when a colleague was incorrectly identified as having scheduled one of their events, they did not seem to realize that they appeared on that person's schedule as well. Consequently, some users came to distrust this feature and ignore it, while others attempted to diagnose it.

\section{Accuracy and trust}

Opinions on the accuracy of attendance predictions were mixed. Some participants found the predictions reasonable, while others said that they seemed overly optimistic or were wrong for some events. One claimed that for his advisor, the predictions "made him seem too faithful" to his calendar. In expressing their degree of trust in Augur's attendance predictions, we found that participants often qualified their ratings with references to uncharacteristic or unforeseen circumstances. For instance, during 
the study period, one participant was injured and out of work for one week, while other participants experienced occasional sick days.

In addition to these unexpected events, participants also experienced disruptions in their schedules from periodic or long-planned events. In one case, a participant had a baby during the latter half of the study. In another example, trust in attendance predictions dropped during the final weeks of classes, when final exams and projects were on the forefront of both students' and professors' minds, with one student observing "During finals week, I'm inferring schedules more than looking at them".

Of course, one of the objectives of Augur is to infer when special events will be attended over conflicting routine schedules. However, the difficulty with these exceptional cases is that schedules were in large part not altered. Augur cannot predict attendance for events that are not present in the calendar. Predictions near the end of the study period thus decreased in value dramatically because, as one professor put it, "at the end of the semester people are skipping things left and right".

In some cases, calendar owners publicized disruptions in their schedules in advance, using "heads-up" emails before the event or mentioning them during meetings. One student complained of a professor's calendar, "If he isn't there at 10:00am then where is he?", implying that although Augur can predict when an event will not be attended, if the calendar is incomplete, it cannot offer an alternate location for that person.

\section{Privacy and impression management}

Participants reported many of the privacy management practices described by Palen [16]. For example, some users renamed appointments to obscure them. Others created appointments to block off undisturbed work time "so you don't look available when you aren't". One student was surprised at the openness of colleague calendars, saying "I looked at someone's calendar and thought 'I wouldn't share that"'. Others were concerned about those mentioned in their own appointments: "I thought about third parties - does that person want their name shared?" In general, however, over half the participants interviewed increased the number of events on their calendar, often adding detail and clarity in case someone viewed their schedule.

One issue with Augur's event matching feature was that incorrect matches could more easily draw attention to event descriptions with parties who had not actually scheduled the same events. In some cases, this could give the identities of third parties involved in those events whose names were in the event descriptions. Provided they had been granted access, participants would normally need to explicitly browse the colleague's calendar to see these events.

In addition, three interviewed participants reported performing tasks specifically to manage the effects of uncertain predictions. For instance, when predictions did not meet user expectations or were decidedly wrong, some participants chose to explore Augur in an attempt to diagnose the errors. Upon noticing that a colleague was incorrectly matched to one of her events, a student reported that she proceeded to look at other calendars for similar errors, including that of the mismatched colleague.

Given that Augur presents the same calendar view to the calendar's owner as it does to any colleagues who might look at it, one participant viewed her own calendar in Augur to confirm that it was showing her events properly. In this case, she corrected typos and removed "Dr." from doctor's appointments after confirming the 
calendar view on Augur. Additionally, she modified her individual meetings with a professor to match his corresponding event exactly, ensuring that they would be recognized as the same event by Augur.

\section{Discussion}

\subsection{Why Was Augur Not Adopted?}

It is clear from our overview of use that in the majority of cases, participants were making more use of the shared calendar functionality of Augur than the predictive features it offered. In addition, use was infrequent and generally failed to displace the existing methods of coordination employed prior to Augur's introduction.

In terms of adoption, Augur suffered from a combination of inertia from existing methods of coordination, barriers to use in terms of accessibility, and maintenance issues with regard to privacy and impression management. While most users expressed an insufficient level of awareness to conduct work with their colleagues, Augur did not provide this information in a form that warranted its adoption over email, office visits, and existing schedule-sharing methods. Though event attendance predictions were designed to be a service supporting students, faculty, and administrative assistants alike, this additional information was not enough for busy professors who were often not in their offices or had little time to spare for navigating a web-based calendar. Judging from their recommendations, adoption may have been improved by porting Augur's interface to a mobile platform and providing one-click bookmarks to frequently browsed calendars.

In the five relationships between faculty members examined during interviews, professors indicated a strong need for improved schedule information throughout the study. Interviews revealed that professors preferred the ease of face-to-face communication over starting a browser, finding the link to Augur, and logging in. One faculty member also suggested a more portable version of Augur that could be used on a phone or PDA, thus freeing her from the need to find a PC in order to use it. While predictive information stood to improve schedule awareness that had been deemed insufficient for work, this potential benefit was outweighed by barriers to use that did not fit the needs of busy, locally mobile professors.

Augur did provide some help to close working relationships and less close relationships with a diminished ability to coordinate unplanned meetings. For close relationships, Augur's additional information occasionally offered value over existing coordination tools, while for less close colleagues, it made up for a lack of existing intuition of each others' schedules. Colleagues with more intermittent, formal working relationships tended to have less use for Augur, as did users with social, highly informal relationships that involved more impromptu coordination.

\subsection{Uncertain Predictions Affect Shared Impressions}

It is clear that shared predictions have the potential to cause concerns over image and privacy. In the case of Augur, participants seemed to be more interested in checking their own attendance predictions than those of their colleagues. For instance, some Augur participants found themselves double-checking their calendars to make sure 
events were properly matched, or diagnosing errors to determine how best to mitigate them. Even those who took no actions to manage these predictions sometimes voiced concerns with them. The benefits of creating and sharing these predictions must outweigh the costs associated with training models and maintaining a desired image. In the case of Augur, only a subset of participants, typically subordinates, seemed to benefit from these facilities, but these benefits depended largely on the participation of others, typically superiors, who received less benefit from the system.

Augur's predictive information could have benefited from more mechanisms for feedback and control, a common concern in ubicomp systems [3]. Sharing of predictions could use the same access control lists used for sharing calendar events, but providing transparency for these predictions is a critical challenge. While participants were told that their attendance information would be used to train the models used by Augur, no direct control over the models was provided. The Bayesian networks and support vector machines underlying Augur's predictions are complex systems that lack a simple, compact means of explaining their results. Mechanisms for allowing users to correct or override these models may provide this control, but users must understand the cause/effect relationships of the feedback they provide.

It is important for designers to realize that forecasting groupware systems which draw their predictions based on past behavior patterns will likely encounter exceptional cases. Conventions such as "heads-up" emails can compensate for some of the coordination problems that may occur, and designers should consider how best to support this practice such that any interested, permitted party can stay informed. Another option is to expose more of the available input to other users so that they may draw their own conclusions, as in MyVine [5].

\subsection{Users Enjoy and Benefit from Calendar Surfing}

One positive design element came from participants' desires to "surf" calendars, a practice that was made easier by Augur's event-matching capability and by most participants' willingness to share their calendars with all Augur users. As elements of organizational learning and socialization were reported as a result of this feature, further research could explore the potential of recommending calendar events from colleagues' schedules where access permissions allow. However, there is certainly a downside in terms of privacy. Not every workplace culture exhibits the openness of an academic research group, and familiarity with potential calendar viewers diminishes as the deployment site grows in size. In less open cultures in large institutions, additional steps may be required to aggregate forecasts among a larger group or to provide different levels of detail for different potential viewers.

\section{Future Work and Conclusions}

The fact that some participants expressed concern over their appearance to others through Augur demonstrates that steps should be taken to ensure that users retain control over this shared image. In recent work, we have examined how users' mental models form over the course of using forecasting groupware tools [18]. We hope to use these results to provide interface design recommendations that can enable better 
interfaces for conveying correct mental models. Overrides could also be incorporated into Augur, allowing users to set their own predicted attendance as needed. While this may be a less accurate picture of a true schedule in some cases, it would allow for the correct representation of exceptional cases and provide a means for one's image to better fit into a particular organizational culture. Similar mechanisms have been suggested in and [2] and [5].

In summary, the predictive capabilities of the Augur system had both positive and negative impacts on the use of what otherwise would have been a traditional groupware calendar system. Inferences about which users had scheduled the same events had the effect of encouraging exploration and organizational learning. Predictive facilities introduced new issues due to their inherent uncertainty, and suggested a need for increased transparency of Augur's reasoning. While attendance predictions provided limited benefit to certain types of working relationships, for some users they were a source of frustration when predictions misrepresented them. Designers should consider lightweight facilities to allow users to manage their appearance through the calendar system, weighing the costs of using these facilities against the benefits to be gained as presence and availability forecasting tools find their way into mainstream applications.

Acknowledgments. Thanks to the Everyday Computing Lab, Gregory Abowd, Jonathan Grudin, Mark Guzdial, and Eric Horvitz for their feedback on this work. This project was funded by NSF CAREER Award \#0092971.

\section{References}

1. Beard, D., Palaniappan, M., Humm, A., Banks, D., Nair, A., Shan, Y.-P.: A Visual Calendar for Scheduling Group Meetings. In: Proc. CSCW'90, pp. 279-290. ACM Press, New York (1990)

2. Begole, J.B., Matsakis, N.E., Tang, J.C.: Lilsys: Sensing Unavailability. In: Proc. CSCW 2004, ACM Press, New York (2004)

3. Bellotti, V., Sellen, A.J.: Design for Privacy in Ubiquitous Computing Environments. In: Proc. ECSCW'93, pp. 77-92. Kluwer, Dordrecht (1993)

4. Fogarty, J., Hudson, S.E., Lai, J.: Examining the Robustness of Sensor-Based Statistical Models of Human Interruptibility. In: Proc. CHI 2004, pp. 207-214 (2004)

5. Fogarty, J., Lai, J., Christensen, J.: Presence versus Availability: The Design and Evaluation of a Context-Aware Communication Client. International Journal of HumanComputer Studies 61(3) (2003)

6. Grudin, J.: Emerging Norms: Feature Constellations Based on Activity Patterns and Incentive Differences, Microsoft Research, Redmond, WA, pp. 1-11 (2001)

7. Grudin, J., Palen, L.: Emerging Groupware Successes in Major Corporations: Studies of Adoption and Adaptation. In: Masuda, T., Tsukamoto, M., Masunaga, Y. (eds.) WWCA 1997. LNCS, vol. 1274, pp. 142-153. Springer, Heidelberg (1997)

8. Hill, R., Begole, J.B.: Activity Rhythm Detection and Modeling. In: Proc. CHI 2003, ACM Press, New York (2003)

9. Horvitz, E., Jacobs, A., Hovel, D.: Attention-Sensitive Alerting. In: Fifteenth Conference on Uncertainty and Artificial Intelligence, pp. 305-313. Morgan Kaufmann, San Francisco (1999) 
10. Horvitz, E., Kadie, C.M., Paek, T., Hovel, D.: Models of Attention in Computing and Communication: From Principles to Applications. Communications of the ACM 46(3)

11. Horvitz, E., Koch, P., Kadie, C.M., Jacobs, A.: Coordinate: Probabilistic Forecasting of Presence and Availability. In: Proc. UAI 2002, pp. 224-233. AAAI Press, California, USA (2002)

12. Hudson, S.E., Fogarty, J., Atkeson, C., Avrahami, D., Forlizzi, J., Kiesler, S., Lee, J., Yang, J.: Predicting Human Interruptibility with Sensors: A Wizard of Oz Feasibility Study. In: Proc. CHI 2003, ACM Press, New York (2003)

13. Kern, N., Antifakos, S., Schiele, B., Schwaninger, A.: A Model for Human Interruptability: Experimental Evaluation and Automatic Estimation from Wearable Sensors. In: ISWC. 8th International Symposium on Wearable Computing (2004)

14. Kraut, R.E., Fish, R.S., Root, R.W., Chalfonte, B.L.: Informal Communication in Organizations: Form, Function, and Technology. In: Oscamp, S., Scacapan, S. (eds.) Human reactions to technology, Sage Publications, Beverly Hills, CA (1990)

15. Mynatt, E.D., Tullio, J.: Inferring calendar event attendance. In: Proc. IUI 2001, pp. 121128. ACM Press, New York (2001)

16. Palen, L.: Social, Individual, and Technological Issues for Groupware Calendar Systems. In: Proc. CHI'99, pp. 17-24. ACM Press, New York (1999)

17. Palen, L., Grudin, J.: Discretionary Adoption of Group Support Software: Lessons from Calendar Applications. In: Munkvold, B.E. (ed.) Implementing Collaboration Technologies in Industry, Springer, Heidelberg (2002)

18. Tullio, J., Dey, A., Chalecki, J., Fogarty, J.: How it Works: A Field Study of NonTechnical Users Interacting with an Intelligent System. In: Proc. CHI 2007 (2007)

19. Tullio, J., Goecks, J., Mynatt, E.D., Nguyen, D.H.: Augmenting Shared Personal Calendars. In: Proc. UIST 2002, pp. 11-20. ACM Press, New York (2002)

20. Whittaker, S., Frohlich, D., Daly-Jones, O.: Informal workplace communication: what is it like and how might we support it? In: Proc. CHI'94, pp. 131-137. ACM Press, New York (1994) 